\title{
Horizontal flight trajectory optimization considering RTA constraints
}

\author{
Alejandro Murrieta-Mendoza, ${ }^{1,}$, Hugo Ruiz ${ }^{2}$, and $\underline{\mathrm{R} u x a n d r a}$ Mihaela Botez ${ }^{3}$ \\ ${ }^{1}$ LARCASE, École de Technologie Supérieure, Université du Québec, Canada \\ ${ }^{2}$ LARCASE, École de Technologie Supérieure, Université du Québec, Canada \\ ${ }^{3}$ LARCASE, École de Technologie Supérieure, Université du Québec, Canada
}

\begin{abstract}
The increasing of flights around the world has led to various problems for the aeronautical industry such as saturated air space and higher levels of fossil fuel consumption. The way in which en-route flights are handled should be improved in order to increase airways' capacity. A solution is to make aircraft to arrive at specific waypoints at a time constraint called Required Time of Arrival (RTA). Fossil fuel brings as a consequence the release of polluting particles to the atmosphere such as carbon dioxide and nitrogen oxides. It is thus desirable to compute the most economical trajectory in terms of fuel burn while fulfilling the RTA constraint. This article proposes a horizontal reference trajectory optimization algorithm based on the Particle Swarm Optimization technique in order to reduce fuel burn while fulfilling the RTA constraint. Results showed that for a flight without RTA constraint, up to $4 \%$ of fuel can be saved comparing against the trajectory of reference. The algorithm was normally able to meet the RTA constrain. However, aggressive RTA constraints might reduce the optimization levels of fuel compared with flights without RTA constraint.
\end{abstract}

\section{Introduction}

Air traffic is expected to grow in the forthcoming years according with market outlooks by Airbus 1 and Boeing2. The geographical places where traffic is expected to have an important growth are those in development: Asia, and Latin America.

The traffic increase has a direct impact on the fossil fuel required to power flights, and thus in the number of polluting particles released to the atmosphere. Among these particles, Carbon dioxide (CO2), Nitrogen Oxides (NOx), and hydrocarbons (HC) are of interest: $\mathrm{CO} 2$ has been of special interest due to its effect on the global warming effect, NOx has been attributed to deplete the ozone layer [1], and $\mathrm{HC}$ cause health problems and reduces the air quality [2].

From an economical perspective, it is expected that government will impose economic measures for pollution avoidance, especially for $\mathrm{CO} 2$. Fossil fuel also represents an important expense for airlines as any reduction on fuel burn could positively impact profit.

\footnotetext{
* Corresponding author: aj70290@ens.etsmtl.ca
} 
Because of these two reasons (pollution and economic consequences), the importance of reducing fuel consumption becomes evident.

By recognizing the impact of fuel to profits, airlines have implemented different measurements to reduce fuel consumption. As discussed in [3], these measurements consists, among others, in improving efficiency by washing dirt off, reduce the use of the auxiliary power, and the aircraft weight reduction by using lighter equipment.

Flight operations might also reduce fuel consumption. According to different studies, in the continental United States airspace [4,5] and in the Turkish airspace [6] aircraft do not fly at their optimal speeds and altitudes. It is thus of interest to provide the aircraft with a more economical reference trajectory.

One of the first improvements in trajectory optimization was in the descent phase. A descent procedure called a Continuous Descent Approach (CDA) was developed. Nevertheless, care must be given to correctly compute the beginning of descent location point to avoid missing the descent leading to a missed approach, which according to [7] it is expensive in terms of fuel consumption. The CDA technique has been implemented at different airports and gave good results [8-11].

Reference trajectory and airspace optimization are of interest to reduce fuel consumption, and to increment the airspace capacity while guaranteeing aircraft separation and safety. Different countries have been working in various programs to improve and optimize the airspace. In this context, the United States are developing the Next Generation of ATM System (NextGen), and Europe has been working with the Single European Sky ATM Research (SESAR).

These programs proposed the Trajectory Based Operations (TBO) or the Intended Based Operations (IBO). By use of these operations, the aircraft would be able to negotiate its optimal reference trajectory with traffic control instead of having an imposed reference trajectory. Future systems intend to use intended 4D trajectories, which means that aircraft position (lat/lon), speed, altitude and Required Time of Arrival (RTA) to a given (or to a set) waypoint will be required.

Reference trajectory optimization could be classified in two main different types: the set of coordinates (waypoints) an aircraft has to follow, which is known as Lateral NAVigation reference trajectory (LNAV) and the set of speeds and altitudes that the aircraft has to follow, which is known as Vertical NAVigation reference trajectory (VNAV). In VNAV, the aircraft can perform changes in altitude (step climbs) in order to find favourable weather or less air resistance (drag force) into the flight in order to reduce fuel consumption [12].

Computing the most efficient trajectory in terms of fuel burn is difficult since the aircraft fuel burn is affected by different parameters such as weather, the change of mass due to fuel consumption, the diversity of combinations available to perform the flight, and the infinite positions where to execute a step-climb.

To compute the most economical trajectory, different algorithms using optimal control have been used as in [13-18] have been implemented. The VNAV reference trajectory for all flight phases has been optimized by using the golden search section and step climbs [19], a search space reduction algorithms were developed in $[20,21]$, and the branch and bound algorithm was implemented in [22], both algorithms were later coupled to reduce the computation time [23]. Genetic Algorithms were also used to find the waypoints where to maximize fuel savings [24].

For the LNAV reference trajectory Classical optimization techniques such as finding the shortest path technique have been used to find the most efficient LNAV trajectory $[25,26]$ a as well as avoiding obstacles in LNAV for general purposes aircraft. [27]. Dijkstra's Metaheuristic algorithms have been successfully used to compute the optimal LNAV trajectory at fixed altitudes [28-30]. 
The objective of this paper is to validate the potential of the Particle Swarm Optimization (PSO) Algorithm in order to find the most economical reference trajectory in LNAV between two points for intercontinental flights. Through this paper, the word "optimal" refers to the trajectory that provides the most economical fuel consumption. This paper is arranged as follows. Firstly, the fuel burn model is described along with the way in which the flight cost is computed. Next, the weather model is explained. Then, the search space and the objective function are presented. Then, the PSO algorithm implementation is detailed, followed by results and conclusions.

\section{Methodology}

\subsection{Fuel Burn Model and Trajectory Cost Computation}

Captions The fuel burn is computed using a Performance DataBase (PDB) which was developed using flight test data. The PDB is a set of tables that provide for given inputs a certain output. The inputs for the cruise phase table are the Aircraft weight, Speed (Mach or IAS), altitude, and the International Standard Atmosphere Temperature Deviation (ISA_DEV). The ISA_DEV gives the difference between the real temperature and the temperature provided by the standard atmosphere model The PDB gives as result the fuel flow for those flight conditions.

Use of a PDB requires a procedure to compute the flight fuel consumption. A complete methodology to compute the flight cost from a database by considering all flight phases such as climb, acceleration, cruise, descent, and deceleration was discussed in [29, 30]. In this paper, only the procedure to compute the fuel consumption in the cruise phase is briefly explained.

Because of the fact that not each required input value is available to provide the required output (fuel flow for the cruise phase), interpolations are required. For these interpolations to be performed, first the lower and the upper bounds surrounding the required values are identified, which is done for each input. A set of linear interpolations are executed to compute the required output from the identified inputs. Figure 1 provides a scheme of the interpolations required to obtain a given fuel flow for a flight condition.

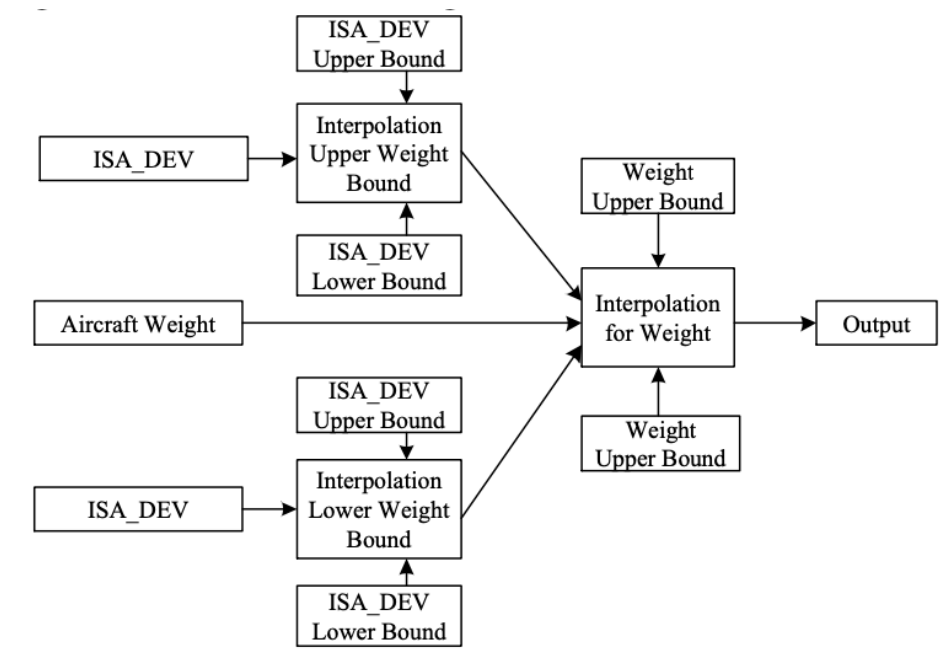

Figure. 1.Interpolation Schema 
In order to obtain the fuel burn for a given cruise distance, the trajectory is divided in different segments. The obtained fuel flow is multiplied by the flight time required to travel a given segment as shown in Eq. (1).

$$
F B_{\text {seg }}=F F_{\text {seg }} * \text { Flight Time }
$$

As fuel is burned during flight, the aircraft mass diminishes over time. This fuel burned should be reduced from the total aircraft weight every time the aircraft flies a pre-defined distance. This distance is important since the accuracy of results depends on how often the aircraft mass is updated, the more often the mass is updated, the more accurate the fuel consumption computation is. However, updating the aircraft mass too often will have as a consequence the execution of a high number of interpolations, that might increase the computation time. These computations are performed until the last segment is flown.

The flight time to travel a given distance must be computed using the Ground Speed (GS), which takes into account wind effects as shown in Eq (2), not the True Air Speed (TAS) or the Indicated Mach number.

$$
G S=T A S \pm \text { Wind Speed }
$$

\subsection{Weather Data}

Weather data was obtained from the open weather data forecast generated daily by Weather Canada. These data are provided under the form of a grid that contains the weather information for all over the world for different altitudes at different hours. This grid is provided with a resolution of $0.6 \mathrm{~km} \times 0.6 \mathrm{~km}$.

A detailed method that process the weather information and computes the ground speed can be found in [31]. A brief description on the way to obtain the weather information is described. Firstly, the 4 points of the grid surrounding the aircraft are identified. Then, the four points are interpolated for the required altitude and time. Finally, a bilinear interpolation can be executed to obtain the required weather parameter (e.g. Wind, Temperature) at the aircraft position, and time. Figure 2 schematically shows the steps, and the interpolations followed to compute the weather information before executing bi-linear interpolations.

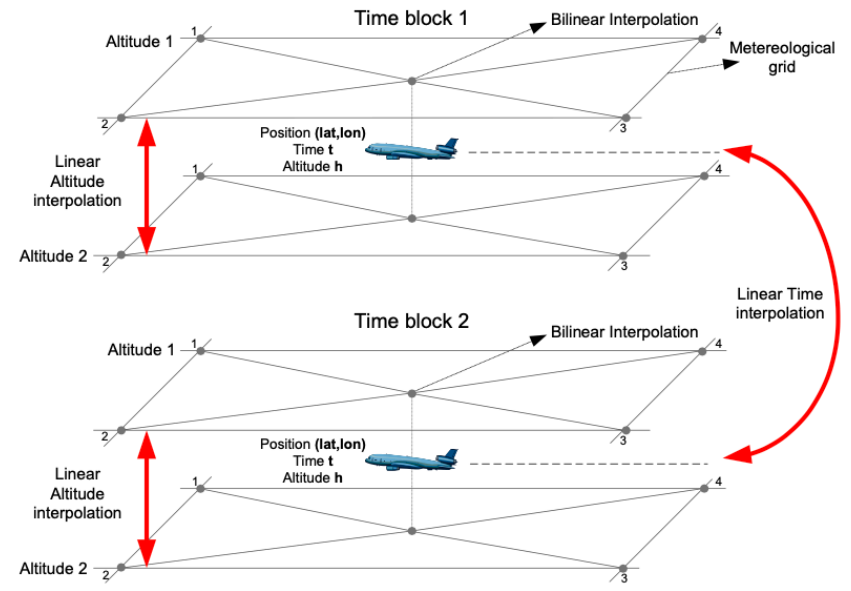

Figure. 2.Weather Interpolations 


\subsection{Search Space}

The search space can be modelled as a weighted graph where edges (E) connect nodes (V). As the study of interest concerns the cruise phase, the first waypoint is the Top of Climb (TOC) and the last waypoint is the Top of Descent (TOD).

In Figure 3, every "node" is considered as a possible waypoint where the aircraft can fly to. The weight expressed in every vertex corresponds to the cost required to reach the connected node.

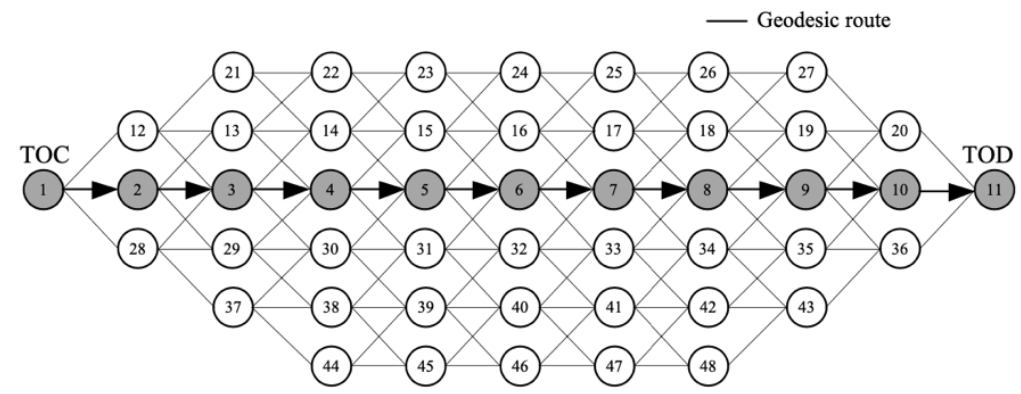

Figure. 3.Lateral Reference Trajectory Asymmetric Graph

\subsection{The Optimal Solution and the Objective Function}

The algorithm aims to minimize Eq (3).

$$
\text { Flight Cost }=\text { Fuel Burn }+ \text { abs(RTA_error }) * R T A \_c o s t
$$

where RTA_error is the difference between the arrival time and the RTA constraint. RTA_cost is the unit cost of missing the RTA. A flight that misses the RTA constraint becomes more expensive, as it is penalized with an extra cost (RTA_cost), thus it is most likely to be rejected by the algorithm. This cost increase is given by the time difference between the arrival time, and the RTA constraint multiplied by a user pre-defined RTA_cost. The larger the difference between the arrival time and the required RTA is, the more expensive the flight cost becomes. It should be noted that this artificial flight cost is an artificial cost used only to reject trajectories that do not fulfil the RTA constraint.".

\section{The Algorithm}

\subsection{The Particle Optimization Algorithm}

The objective is to find the combinations of waypoints from the $\mathrm{ToC}$ to the ToD that would provide the most economical way to connect them. For this is a combinatory problem resolution, it has been proved that the metaheuristic PSO algorithm performs well in finding very good solutions. The PSO, first described in [32], mimics the behaviour of social animals which follow a leader during their activities such as flocks of birds or fish schools.

The PSO algorithm is iterative. At every iteration, particles are modified in order to find new solutions within the search space. These particles are influenced by their own position, a global leader's positions and another particle's positions. Each particle in the PSO algorithm can be define as a set of waypoints from ToC to ToD, or in other words, as a complete trajectory. The equations that model these influences are shown in Eq (4) and Eq (5). 


$$
\begin{gathered}
D_{i}(k+1)=\omega R_{\omega} D_{i}(k)+c_{1} R_{1}\left(B_{l}-X_{i}(k)\right)+c_{2} R_{2}\left(B_{g}-X_{i}(k)\right) \\
X_{i}(k+1)=X_{i}(k)+D_{i}(k+1)
\end{gathered}
$$

where $i$ is a trajectory, and $k$ is the current iteration. D is the particle's displacement associated with the parameter $i$, which indicates if the displacement is in the lateral or vertical reference trajectory. $\mathrm{X}$ is the next particle's location. $B_{z}$ is the best global trajectory position, and $B_{\imath}$ is the best local trajectory position. In order to have an influence in the solution convergence, there are 3 random parameters $R_{\omega}, R_{\imath}$ and $R$, with values between 0 and 1 . The role of these parameters $R$ is to determine the influence on the particle's movement. The parameters $C_{t}$ and $c_{2}$ are positive constants provided by the user, which influence the particle movement in reference to the $B_{s}, B$. The parameter $\omega$ refers to the aircraft's inertia and it is set by user. The inertia represents the particles tendency to keep the same direction on the search space.

\subsection{The LNAV Optimization Algorithm}

The steps required to compute the optimal LNAV reference trajectory is as follows

Step 1: Random Trajectory Generation. Different clusters with a pre-defined number of trajectories are randomly generated.

Step 2: Flight Cost Evaluation and Selection. All trajectories are evaluated. The most economical is set as the current global leader. The most economical trajectory of each cluster is defined as a local leader.

Step 3: Random Vectors Generation. R vectors are randomly generated.

Step 4: Displacement Computation. The displacement for every single trajectory is computed with Eq (4) and the vectors generated in Step 3. Displacement for this work consists on the set of waypoints to follow and the Mach Speed.

Step 5: New position Computation. The new particle's position (reference trajectory) is updated with the computed displacement in Step 4 using Eq (5).

Step 6: New trajectories evaluation and leaders updated. The flight cost of every trajectory is evaluated. The most economical trajectory is defined as the global leader particle and the most economical trajectory of every cluster is defined as a local leader. Step 7: Stopping criteria. The algorithm stops if a given number of iterations is reached. Otherwise, it returns to step 3.

\section{Results}

The fuel saving potential of the trajectories provide by the algorithm was evaluated for a two engine, wide body aircraft with a mass of $125000 \mathrm{~kg}$ at the ToC and a fixed cruise altitude of $34,000 \mathrm{ft}$. It was considered that the aircraft is flying under the TBO concept, free to fly anywhere in the search space. All flights were intercontinental flights. Optimized flights are compared with their geodesic trajectories (shortest paths in a sphere).

Two sets of LNAV result are presented, one considering only the fuel optimization potential and the other one the optimization with a RTA constraint. 


\subsection{LNAV Optimization}

The first set of results aim to validate the potential of the PSO algorithm to reduce fuel burn. Six different flights: Montreal to Innsbruck (YUL - INS), Montreal to Grand Canary Island (YUL - LEP), Montreal to London (YUL - LON), Vancouver to Grand Canary Island (YVR - LPS), and Los Angeles to Innsbruck (LAX - INS). Fuel saving results are shown in Figure 4.

It can be seen from Figure 4 that all flights gave better results than the geodesic trajectory of reference. It can also be seen that the longest trajectories (YVR - LPS and LAX - INS) gave more fuel savings than the other routes. This is because the longer the trajectories the algorithm was, depending the case, able to benefit from tail winds or avoid head winds for a longer period.

\subsection{LNAV Optimization with RTA constraint}

The second set of results aimed to observe the optimization savings when a RTA constraint was set. In this case, the RTA allowed error was of +/- 30 seconds, and the Mach numbers that the algorithm could select were between 0.62 and 0.8 . The same flight cases were evaluated as the ones in Section 4.1. The RTA constrain was selected quit aggressive by setting it to be the same value as the reference trajectory. Fuel saving results are shown in Figure 5.

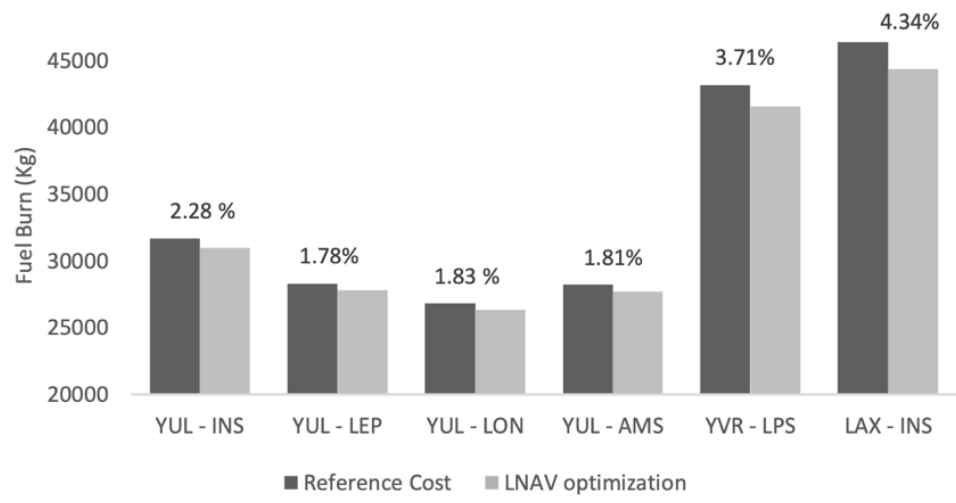

Figure. 4. Flight Savings without a RTA Constraint.

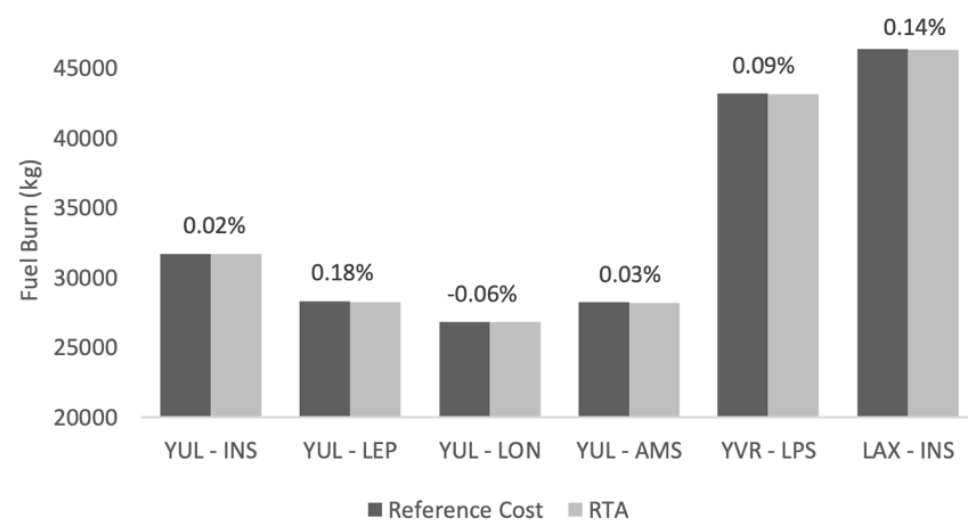

Figure. 5. Flight Savings with a RTA Constraint. 
It can be seen from Figure 5 that by imposing an aggressive RTA constraint, the optimization is reduced to practically zero. Even the flight YUL - LON recorded a more expensive flight than the reference flight.

The RTA constraint information is shown in Table 1 where in almost all cases, the RTA constraint was met. The only exception were the flights YVR - LPR and LAX - INS which are the longest ones. It can be seen that the first 4 flights (with similar distances) were all close to the RTA limit. The highest error for the RTA was given by the LAX - INS flight which was also the longest flight. This result was due to the fact that the algorithm only generates one Mach number during flight. To reduce this error, the algorithm should be able to change aircraft speed during flight.

Table 1. RTA performance.

\begin{tabular}{|c|c|c|c|c|c|}
\hline Flight & $\begin{array}{c}\text { TOC } \\
\text { Time }(\mathrm{h})\end{array}$ & $\begin{array}{c}\text { TOD RTA } \\
(\mathrm{h})\end{array}$ & $\begin{array}{c}\text { Optimal } \\
\text { Arrival }(\mathrm{h})\end{array}$ & $\begin{array}{c}\text { Error } \\
(\mathrm{s})\end{array}$ & $\begin{array}{c}\text { Flight } \\
\text { Time }(\mathrm{h})\end{array}$ \\
\hline YUL - INS & 4 & 11.742 & 11.741 & 28 & 7.7 \\
\hline YUL - LEP & 4 & 10.812 & 10.813 & 25 & 6.8 \\
\hline YUL - LON & 4 & 10.492 & 10.495 & 23 & 6.5 \\
\hline YUL - AMS & 4 & 10.840 & 10.838 & 25 & 6.8 \\
\hline YVR - LPS & 4 & 14.745 & 14.736 & 39 & 10.7 \\
\hline LAX - INS & 4 & 15.707 & 15.695 & 42 & 11.7 \\
\hline
\end{tabular}

\section{Conclusion}

The LNAV optimization algorithm based on PSO was able to find combinations of waypoints and a Mach number that would reduce the flight consumption up to $4 \%$ with respect to the reference trajectory. This fact could potentially lead to a reduction of polluting emissions and the flight cost.

The algorithm should be able to generate more than one Mach number per flight in order to meet the RTA constraint and to save fuel for long-haul flights. It was observed that by imposing aggressive RTAs, the optimization opportunities might be reduced.

\section{Acknowledgements}

This research was conducted at the Laboratory of Applied Research in Active Controls, Avionics and Aeroservoelasticity (LARCASE) within the frame of the global project "Optimized Descent and Cruise" with funds from the Business-led Network of Centers of Excellence Green Aviation Research \& Development Network (GARDN). For more information please visit http://larcase.etsmtl.ca. The authors would like to thank Rex Haygate, Dominique Labour and Yvan Blondeau from CMC- Electronics - Esterline, and Oscar Carranza from LARCASE. The authors would also like to thank CONACYT in Mexico and the FQRNT in Quebec, Canada. 


\section{References}

1. P. J. Crutzen. "The influence of nitrogen oxides on the atmospheric ozone content," Q.J.R. Meteorol. Soc Vol. 96, No. 408, (1970), pp. 320-325. doi: 10.1002/qj.49709640815

2. E. Robinson. "Hydrocarbons in the atmosphere," Pure and applied geophysics Vol. 116, No. 2-3, (1978), pp. 372-384. doi: 10.1007/bf01636892

3. D. McConnachie, C. Wollersheim, and R. J. Hansman. "The Impact of Fuel Price on Airline Fuel Efficiency and Operations," 2013 Aviation Technology, Integration, and Operations Conference. American Institute of Aeronautics and Astronautics, (2013).

4. L. Jensen, J. R. Hansman, J. Venuti, and T. Reynolds. "Commercial Airline Altitude Optimization Strategies for Reduced Cruise Fuel Consumption," 14th AIAA Aviation Technology, Integration, and Operations Conference. American Institute of Aeronautics and Astronautics, (2014).

5. L. Jensen, J. R. Hansman, J. C. Venuti, and T. Reynolds. "Commercial Airline Speed Optimization Strategies for Reduced Cruise Fuel Consumption," 2013 Aviation Technology, Integration, and Operations Conference. American Institute of Aeronautics and Astronautics, Los Angeles, USA, (2013).

6. E. T. Turgut, M. Cavcar, O. Usanmaz, A. O. Canarslanlar, T. Dogeroglu, K. Armutlu, and O. D. Yay. "Fuel flow analysis for the cruise phase of commercial aircraft on domestic routes," Aerospace Science and Technology Vol. 37, No. 0, (2014), pp. 1-9. doi: http://dx.doi.org/10.1016/j.ast.2014.04.012

7. A. Murrieta-Mendoza, R. M. Botez, and S. Ford. "New method to compute the missed approach fuel consumption and its emissions," The Aeronautical Journal Vol. 120, No. 1228, (2016), p. 18. doi: http://dx.doi.org/10.1017/aer.2016.37

8. T. Kwok-On, B. Daniel, and W. Anthony. "Development of Continuous Descent Arrival (CDA) Procedures for Dual-Runway Operations at Houston Intercontinental," 6th AIAA Aviation Technology, Integration and Operations Conference (ATIO). American Institute of Aeronautics and Astronautics, (2006).

9. I. De Lépinay, D. Dimitriu, and A. Melrose. "Environmental impact assessment of Continuous Descent Approaches at Manchester and Bucharest airports," 35th International Congress and Exposition on Noise Control Engineering, INTER- NOISE 2006. Vol. 6, Institute of Noise Control Engineering of the USA -, Honolulu, HI, United states, (2006), pp. 3856-3863.

10. T. Kwok-On, W. Anthony, and B. John. "Continuous Descent Approach Procedure Development for Noise Abatement Tests at Louisville International Airport, KY," AIAA's 3rd Annual Aviation Technology, Integration, and Operations (ATIO) Forum. American Institute of Aeronautics and Astronautics, (2003).

11. D. Novak, T. Buckai, and T. Dadisic. "Development, Design and Flight Test Evaluation of Continuous Descent Approach Procedure in FIR Zagreb," Scientific Journal on Traffic and Transportation Research Vol. 21, No. 5, (2009), pp. 319- 329. doi: http://dx.doi.org/10.7307/ptt.v21i5.247

12. J. A. Lovegren. "Estimation of potential aircraft fuel burn reduction in cruise via speed and altitude optimization strategies." Massachusetts Institute of Technology (2011), p. 97. 
13. P. Bonami, A. Olivares, M. Soler, and E. Staffetti. "Multiphase Mixed-Integer Optimal Control Approach to Aircraft Trajectory Optimization," Journal of Guidance, Control, and Dynamics Vol. 36, No. 5, (2014), pp. 1267-1277. doi: http://dx.doi.org/10.2514/1.60492

14. J. A. Cobano, D. Alejo, G. Heredia, and A. Ollero. "4D Trajectory Planning in ATM With an Anytime Stochastic Approach," Proceedings of the 3rd International Conference on Application and Theory of Automation in Command and Control Systems. ACM, Naples, Italy, (2013).

15. B. Korn, H. Helmke, and A. Kuenz. "4D Trajectory Managemetn In The Extended TMA: Coupling AMAN And 4D FMS For Optimized Approach Trajectoreis," 25th Congress of International Council of the Aeronautical Sciences. Vol. Paper ICAS 20068.8.2 Optimage Ltd, Hamburg, Germany, (2006).

16. M. Soler-Arnedo, M. Hansen, and B. Zou. "Contrail Sensitive 4D Trajectory Planning with Flight Level Allocation Using Multiphase Mixed-Integer Optimal Control," AIAA Guidance, Navigation, and Control (GNC) Conference. American Institute of Aeronautics and Astronautics, (2013).

17. C. Tsotskas, T. Kipouros, and M. Savill. "Biobjective Optimisation of Preliminary Aircraft Trajectories," Evolutionary Multi-Criterion Optimization. Vol. 7811, Springer Berlin Heidelberg, (2013), pp. 741-755.

18. A. Valenzuela, and D. Rivas. "Optimization of Aircraft Cruise Procedures Using Discrete Trajectory Patterns," Journal of Aircraft Vol. 51, No. 5, (2014), pp. 16321640. doi: http://dx.doi.org/10.2514/1.C032041

19. R. S. Félix-Patrón, R. M. Botez, and D. Labour. "New Altitude Optimisation Algorithm for the Flight Management System CMA-9000 Improvement on the A310 and L-1011 Aircraft," The Aeronautical Journal Vol. 117, No. 1194, (2013), pp. 787-805.

20. J. Gagné, A. Murrieta-Mendoza, R. Botez, and D. Labour. "New Method for Aircraft Fuel Saving Using Flight Management System and Its Validation on the L- 1011 aircraft," 2013 Aviation Technology, Integration, and Operations Conference, (2013). doi: http://dx.doi.org/10.2514/6.2013-4290

21. A. Murrieta-Mendoza, and R. M. Botez. "Vertical Navigation Trajectory Optimization Algorithm For A Commercial Aircraft," AIAA/3AF Aircraft Noise and Emissions Reduction Symposium, (2014). doi: http://dx.doi.org/10.2514/6.2014- 3019

22. A. Murrieta-Mendoza, B. Beuze, L. Ternisien, and R. M. Botez. "New reference trajectory optimization algorithm for a flight management system inspired in beam search," Chinese Journal of Aeronautics, (2017). doi: http://dx.doi.org/10.1016/j.cja.2017.06.006

23. A. Murrieta-Mendoza, L. Ternisien, B. Beuze, and R. M. Botez. "Aircraft Vertical Route Optimization by Beam Search and Initial Search Space Reduction," Journal of Aerospace Information Systems Vol. 15, No. 3, (2018), pp. 157-171.doi: http://dx.doi.org/10.2514/1.i010561

24. A. Murrieta-Mendoza, R. S. Félix-Patrón, and R. M. Botez. "Flight Altitude Optimization Using Genetic Algorithms Considering Climb and Descent Costs in Cruise with Flight Plan Information," SAE 2015 AeroTech Congress \& Exhibition. SAE International, Seattle, USA, (2015), p. 9.

25. A. Murrieta-Mendoza, and R. M. Botez. "Lateral Navigation Optimization Considering Winds And Temperatures For Fixed Altitude Cruise Using The Dijkstra's Algorithm," International Mechanical Engineering Congress \& Exposition. Montreal, Canada, (2014). 
26. A. Murrieta-Mendoza, C. Romain, and R. M. Botez. "Commercial Aircraft Lateral Flight Reference Trajectory Optimization," 20th IFAC Symposium on Automatic Control in Aerospace ACA 2016 Vol. 49, No. 17, (2016), pp. 1-6. doi: http://dx.doi.org/10.1016/j.ifacol.2016.09.001

27. E. Rippel, A. Bar-Gill, and N. Shimkin. "Fast Graph-Search Algorithms for GeneralAviation Flight Trajectory Generation," Journal of Guidance, Control, and Dynamics Vol. 28, No. 4, (2005), pp. 801-811.doi: http://dx.doi.org/10.2514/1.7370

28. R. S. Félix-Patrón, A. Kessaci, and R. Botez. "Horizontal Flight Trajectories Optimisation for Commercial Aircraft Through a Flight Management System " The Aeronautical Journal Vol. 118, No. 1210, (2014), p. 20.

29. A. Murrieta Mendoza, A. Bunel, and R. Botez. "Aircraft Lateral Flight Optimization Using Artificial Bees Colony," International Conference on Air Transport INAIR 2015. Amsterdam, the Netherlands, (2015).

30. A. Murrieta-Mendoza, A. Hamy, and R. M. Botez. "Lateral Reference Trajectory Algorithm Using Ant Colony Optimization," 16th AIAA Aviation Technology, Integration, and Operations Conference. American Institute of Aeronautics and Astronautics, Washington, D.C., (2016).

31. A. Murrieta-Mendoza. "Vertical and Lateral Flight Optimization Algorithm and Missed Approach Cost Calculation.." Vol. Master, École de Technologie Supérieure, Montreal, (2013), p. 114.

32. J. Kennedy and R. Eberhart, "Particle swarm optimization," Proceedings of ICNN'95 International Conference on Neural Networks, Perth, WA, Australia, 1995, pp. 19421948 Vol.4. doi: http://10.1109/ICNN.1995.488968 